\title{
ARTIKEL
}

ANDRZEJ DENKA

Poznań

\section{VATERSEHNSUCHT. BOTHO STRAUß ERZÄHLT SICH SEINE HERKUNFT}

\begin{abstract}
Longing for a father. Botho Strauß tells himself about his own 'Origin'
Botho Strauß (b. 1944), German playwright, novelist and essayist, devotes his book Herkunft [Origin] (2014) to a subtle portrait of his slightly underestimated father, who died in 1971. This sample of prose is typical of Strauss as it encompasses meditative descriptions, disquisitions, aphorisms and narrative fragments. This narration contains numerous biographical details about his father, as well as his mother and the writer himself, and it tells us a lot about his youth and cultural maturation. Strauss' hometown, Bad Ems, provides a certain topographic point of reference here. This is a highly personal and emotional text which simultaneously exhibits all esthetic properties that characterize Strauss' style. This text is also about the way different sensory stimuli incite our memory and how difficult it is to find a literary form adequate to reconstruct memory.
\end{abstract}

KEY WORDS: Botho Strauß, “Origin”, fatherhood, memory, biography, Bad Ems

\section{Anlässe, Anläufe, Anliegen}

Botho Strauß hat ein Meisterwerk geschrieben. Nicht nur, weil der Jahrzehnte lang als nicht unumstritten geltende Dramaturg, Essayist und Prosaiker Botho Strauß (geb. 1944) nahezu einstimmig Anerkennung findet in den Kritiken zum Prosaband Herkunft (2014) und man es endlich - und ohne Vorbehalte - sagen darf: „Botho Strauß ist unser wichtigster Autor (...)“ (Mangold, 2014), sondern weil das Buch tatsächlich außergewöhnlich ist. Bei der Lektüre läuft einem ein Schauer über den Rücken, weil die emotionale Entblößung alles übertrifft, was den Strauß-Lesern aus anderen Werken dieses Autors bereits geläufig ist: brillante Intelligenz, einzigartige Sprachgewandtheit, denkerischer Eigensinn des Bocksgesang-Autors.

Der 70jährige Botho Strauß erzählt in Herkunft über seinen Vater, vielmehr: er erschafft sich seinen Vater aus den Bruchstücken der Erinnerung, verfolgt die 
oftmals vagen Spuren des Gedächtnisses, was sich zugleich in einer ästhetischen Suche nach der angemessenen Form niederschlägt. Nicht zuletzt ergibt sich so ein poetologischer Text, eine Reflexion der höheren Ordnung (Beobachtung zweiter Ordnung, Luhmann) an der Schnittstelle des psychologischen Erlebnisses und der Kunst. Es ist ein inkohärenter, mitunter brüchiger Text, Narrationssplitter sind es, aus denen die Biographie des Vaters sich zusammensetzt. Diese Biographie zu rekonstruieren, bedarf einiger Mühe, genauso wie es einiger Mühe bedarf, den Anlass und das eigentliche Anliegen, hier das als unmittelbar berichtete Geschehen aus dem Jahr 1997, zu erfassen. Volker Hage (2014) verweist auf die 1990 entstandene, unveröffentlichte Skizze zum 100. Geburtstag des Vaters von Botho Strauß. Dieses Datum wird auch in Herkunft verzeichnet: „Heute, am 9. April 1990, hätte der Vater seinen hundertsten Geburtstag gefeiert.“ (H, S. 4) Offensichtlich war das einer der Anläufe für die Aufgabe der Erinnerung, die jedoch noch eines weiteren Anlasses bedurfte - der Auflösung des elterlichen Haushalts 1997: „Morgen wird die Wohnung der Eltern aufgelöst. Morgen wird meine Kindheit entrümpelt.“ (H, S. 43) In der ausgearbeiteten Form eines Buches aber konnte die Erinnerung erst 2014 bewältigt werden. Diese Anläufe und Anlässe werden im Text reflektiert, fließen in den Suchprozess des Schreibens ein. Das erzählende, konstruierende Subjekt ist Botho Strauß selbst, und diese Gleichsetzung soll keine Vereinfachung sein (das Problem des Autobiographischen in seiner Prosa bedürfte freilich einer gesonderten Auslegung). Gilt Herkunft vordergründig dem Vater, so geht der eigentliche Anlass doch von der Mutter aus, die wegen des fortgeschrittenen Alters nicht mehr alleine in der Wohnung bleiben kann.

Mein Garten dort unten, sagte die Mutter zuweilen. (...), Hier habe ich nun so viele Jahre gesessen und immer hinunter auf die Straße und den Park gesehen.

Genauer gesagt: Von der Mitte des vergangenen Jahrhunderts bis bald zu seinem Ende. Seit über zweiundvierzig Jahren gab es Ems, Römerstraße achtzehn. Nun soll ich anrufen im ,Staatsbad', unserem Mietherrn, um die Wohnung zu kündigen. Die Mutter zieht ins Altersheim.

Am letzten Abend zuhause erinnerten wir uns an den Einzug im Winter fünfundfünfzig (...). (H., S. 49-50) [Hervorhebung A.D.]

Mühsam lassen sich die Daten rekonstruieren. 1955 zogen Botho Strauß' Eltern nach Bad Ems; die Stadt ist der topographische Dreh- und Angelpunkt, Ort des Geschehens, ein handfester Anhaltspunkt für die Rekonstruktion des Textinhalts. 1955 wurde Strauß in Bad Ems in einer Schule angemeldet (H, S. 48, S. 50). Auf die biographischen Angaben der Sekundärliteratur zu Botho Strauß ist allerdings kein Verlass, sie sind lückenhaft. Das (Auto-)Biographische - vom medienscheuen Autor streng als das Persönliche geschützt - sickert aus verschiedenen Prosawerken durch (z.B. Paare, Passanten, 1981; Die Fehler des Kopisten, 1997). Wer Strauß' Prosawerke kennt, fügt sich die Details zusammen, er kennt seine Biographie, nicht nur seine geistige Biographie, sondern auch viele biographische Fakten (Hage, 1987, 
S. 208; Eckhoff, 1999, S. 4-7). Mit Herkunft hat Botho Strauß nun tatsächlich etwas Biographisches geschrieben: eine Biographie seines Vaters, und niemand hat Zweifel, es ist literarische Prosa, Reflexionsprosa, Meisterwerkprosa. Die literaturtheoretische Differenzierung zwischen der Erzählzeit und der erzählten Zeit ist in Bezug auf diesen Text nur als vorläufiges, behelfsmäßiges Konstrukt zu betrachten. Fügt man sich das konkrete Geschehen zusammen, das als erzählte Zeit bezeichnet werden kann, so ergibt sich daraus es hier tatsächlich der erste Anordnungsversuch, ein Erzählrahmen. Es wird eine kurze Zeit, ein Tag, eine Stunde, ein Augenblick im Jahre 1997 erzählt. Eine Rezensentin schreibt vom Jahr 1996 (Arend, 2014) aber der halbwegs gute Mathematiker wird 1955 (Tag des Umzugs der Familie Strauß nach Bad Ems) und 42 (so viele Jahre lebte die Mutter dort) zusammenzählen können und kommt auf das Jahr 1997. Aber halten wir uns nicht an Lappalien, denn nicht das lineare Erzählen gilt hier als Prinzip, sondern eher Diskontinuitäten und Brüche, die der Autor bewusst in Rechnung stellt. Fassen wir zusammen: Aus der Perspektive einiger Stunden an einem Tag (wahrscheinlich) des Jahres 1997 fügt sich das erzählende und konstruierende Subjekt, Botho Strauß, die Biographie des 1890 geborenen und 1971 verstorbenen Vaters, Dr. Eduard Strauß (der Name fällt im Buch nicht, vgl. hingegen Pabst, 2014 bzw. das Stichwort Eduard Strauss bei Wikipedia), zusammen. Doch passt in diese erzählte Zeit weitaus mehr hinein als Daten und Fakten aus dem Leben des Vaters. Erzählt werden auch die Kindheit des Erzählers, seine Jugendzeit, Erlebnisse in der Schule, Erlebnisse mit Freunden, Reminiszenzen aus Lektüren, aus Medien (Comics, Fernsehen), erste Liebe(-n), Faszinationen für Musik, Film, Theater, ebenso Details aus Topographie und Geschichte der Stadt Bad Ems.

\section{Der Vater - ein Porträt}

Die biographischen Fakten werden nicht linear geboten, dennoch verbinden sie sich zu einem Curriculum Vitae. Der von Botho Strauß ,erzählte' Vater zog als Vierundzwanzigjähriger in den Ersten Weltkrieg und erlitt eine Verwundung im Gesicht. Berichtet wird von ,[einem] Geschoß, [einem] Projektil mit der abgebogenen Spitze, das in einer Gefechtsnacht 1916 oberhalb der Nasenwurzel die Stirnwand durchschlug und das linke Auge zerstörte" $(\mathrm{H}, \mathrm{S}$. 15), später ist auch noch einmal von „zerschossener Stirn“ die Rede (H, S. 19). Pabst (2014) vermerkt allerdings - Bezug nehmend auf den Lebenslauf von Eduard Strauß in dessen Dissertation -, dass er die Verwundung bereits „am 24. August 1914“ erlitt. Zu dieser Zeit war Thomas Mann, jener aus der monarchistischen Phase (für die die Betrachtungen eines Unpolitischen (1915-1918) repräsentativ stehen), sein Lieblingsautor (H, S. 11). Der Vater selbst war „kaisertreu“, „deutschnational auf seine Weise“, und blieb es in gewissem Sinne, selbst wenn er nach dem ersten Weltkrieg zum Pazifisten wurde. „Er war ein großer Hasser des Militärs und der Militärs. (Letztere schilderte er mir 
gern als Gestrandete, die nach dem ersten großen Krieg in Kiosken Zigaretten verkaufen mußten).“ (H, S. 180) Da ihm die finanziellen Mittel fehlten, um sein Medizinstudium fortsetzen zu können, studierte er Pharmazie und war später Mitinhaber einer kleinen pharmazeutischen Firma in Naumburg an der Saale. Während der nationalsozialistischen Diktatur veröffentlichte er Broschüren mit Empfehlungen zur Erhaltung der Gesundheit, die dem Sohn einen Schrecken einjagen, enthält doch eine die Empfehlung, sich in der Hitlerjugend sportlich zu betätigen. „Jedenfalls war ich darauf erleichtert, als ich bemerkte, daß in dem Bändchen nirgends sonst vom Völkischen oder von der Volksgesundheit die Rede war." (H, S. 21) Erwähnt wird auch das Buch, das der Vater schrieb: Nicht so früh sterben (1941) (H, S. 12). Dieses Buch wurde 1943 ins Holländische übersetzt und erlebte 1949 eine Neuauflage (Wikipedia, 2016). Nach dem Krieg versuchte der Vater, seinen pharmazeutischen Betrieb in der SBZ weiter zu betreiben, wurde aber enteignet und sogar inhaftiert. Daher flüchtete die Familie in den Westen und suchte dort nach einem besseren Leben, ab 1955 hatte sie einen neuen Wohnsitz: Bad Ems. „Wir sind weggegangen', so hörte ich ihn, ,weil wir nicht wollten, daß du dort zum Kommunisten erzogen wirst'." (H, S. 19) Der Vater versuchte, sein pharmazeutisches Metier fortzuführen, arbeitete für pharmazeutische Firmen, entwickelte neue Medikamente, hatte aber wenig Erfolg. Er verfasste nicht nur Fachartikel, sondern war auch als Verleger aktiv, gab eine kritisch-satirische Monatsschrift, „nach Krausschem Vorbild“ heraus (H, S. 12). Nur schwer fasste er Fuß in der neuen Heimat, alt, eigensinnig, misanthropisch, nicht mehr in seine Epoche passend. 1971 starb er, wahrscheinlich an einem Gehirnschlag. Gefunden hat ihn sein Sohn: „Aus seinem rechten Ohr strömte Blut. Ich wußte, daß es nun zu Ende war, und lehnte mich zu ihm.“ (H, S. 40) Eine lineare Beschreibung, die - auf einige Sätze reduziert - auch in einem Lexikon stehen könnte. Strauß' Text bezweckt aber viel mehr, als nur den Lebenslauf eines unbekannten Pharmazeuten einem breiteren Publikum näher zu bringen.

\section{Ästhetik der Erinnerung}

Die Erinnerung an den Vater, an die eigene Kindheit und Jugendzeit wird begleitet von der ständigen Reflexion, wie Erinnerung überhaupt zustande kommt, wie sie empfunden und wie sie literarisch verarbeitet werden kann. Die Fähigkeit der Erinnerung führt der einst gegen den Vater rebellierende Botho Strauß überhaupt auf den Vater zurück: „Nie hätte ich mich irgendeines Geschehens erinnert ohne deine Schule der Erinnerung. Alles was war, wurde überhaupt Gewesenes durch dich." (H, S. 15). Strauß geht beim Erinnern induktiv vor. Das Erinnern zeigt sich somit unvermittelt, ,authentisch'. Kein fertiges Bild, sondern Einzelheiten, Farben, Stimuli: „[d]ie Schritte, die Geräusche, die Stimme des Vaters im Flur“, „Kaffeegeruch“, „[d]ie frischen Brötchen im Beutel““,dunkle Morgen im späten November“, „mein 
dunkel-blauer, viel zu langer Popelinemantel“, „die ersten Fernsehbilder in Schwarzweiß“" (H, S. 21). So bahnt sich Erinnerung an. Bevor das fertige Bild zum Tragen kommt, muss eine Vorarbeit geleistet, muss die Grundierung geschaffen werden.

Jedes Bild, noch das flüchtigste, war die frühe Einlage in ein Depot von Sinnesreizen, die wertbeständig und hochverzinst dem Alter einen guten Ertrag an Gedächtnis bringen. Die Erweiterung eines Horizonts besteht nicht selten darin, daß sich einem das Gewesene öffnet. Nur auf dem Feld der Erinnerung kann man noch expandieren, reicher werden, zunehmen. (H, S. 21-22)

Ein zufällig aus dem Radio wahrgenommenes Klavierstück von Josef Suk weckt den Wunsch, die ,(...) Mutter einmal in ihren schönsten verführerischen MädchenKleidern tanzen [zu sehen] auf Festen und Empfängen“" (H, S. 34). So konkludiert Strauß, dass die Verlustgefühle im Besonderen das Erinnern antreiben: „Das Gedächtnis ist eine Variable der Sehnsucht, so daß Fernweh und Heimweh, Erwartung und Erinnerung in ein und demselben ,Enzym‘ des Unerreichlichen symmetrisch angeordnet sind." (H, S. 35)

Diese emotionalen Momente haften jeder Erinnerung an, so dass der Prozess des sich Erinnerns selbst zum poetologischen Credo, zum ästhetischen Manifest erklärt wird:

Fügt sich Erinnerung, so schwindet sie schon. Kontinuität der Darstellung, der Erzählung ist dem rohen, unberechenbaren Affekt, dem Anfall oder Ansprung von ,verlorener Zeit', etwas durchaus Unangemessenes. Daher wird immer der Roman am überzeugendsten das Gewesene, das eigentlich unfaßlich ist, gut fügen und kunstvoll ausbreiten. Aber nur das Gedicht kann der eruptive Akt der Erinnerung selber sein, der sich gedächtnis-erregend auf den Leser überträgt. (H, S. 89) [Hervorhebungen A.D.]

Die Erinnerung ist vage, flüchtig, in ihrem eigentlichen Wesen kaum vermittelbar. So ist die Darstellung der Erinnerung mit ihren zahlreichen Affekten in der Prosa nur eingeschränkt möglich. Der Roman vermag immerhin in seiner epischen Breite das „Gewesene“ komplementär und ästhetisch zu schildern: „kunstvoll“. Kunst bedeutet nicht unbedingt Künstlichkeit, vielmehr geht es um einen Filter. Insofern wäre der Roman vor allem eine Beobachtung zweiter Ordnung. Die vermittelte, geordnete und zu einem Ganzen gefügte Erinnerung (sprich: beobachtete Erinnerung) ist keine primäre Erinnerung mehr, es ist eine Reflexion zweiten Grades. So wie (nach Niklas Luhmann) die Kommunikationen andere Kommunikationen beobachten, beobachten die Gedanken andere Gedanken. Wir sind uns nicht bewusst, dass wir einen Gedanken gefasst haben, solange wir nicht mit einem anderen Gedanken Bezug nehmen auf diesen vorangegangenen (Sill, 2001, S. 50). Botho Strauß bringt es auf den Punkt: „Wer seine Erinnerungen erzählt, befindet sich nicht im Zustand der Erinnerung." (H, S. 62)

Das Gedicht im hier gemeinten Sinne entspricht hingegen der autopoiesis von Luhmann, also dem Grundsatz, der besagt, dass das System sein eigenes Werk sei, 
es geht also um die „Produktion des Systems durch sich selber“ (Sill, 2001, S. 43). Das Gedicht - als der ,eruptive Akt der Erinnerung“ (H, S. 89) - konstruiert sich selbst. Man könnte hier die Frage stellen, warum Strauß sich nicht der lyrischen Form bedient, wenn er diese für angemessener erachtet, Erinnerung wiederzugeben? Obwohl auch Gedichte in sein Euvre fallen (etwa das umstrittene, 80 Seiten lange Gedicht Diese Erinnerung an einen, der nur einen Tag zu Gast war, 1985), bedient sich der Autor, der oft als Dichter bezeichnet wird und sich offensichtlich auch selbst für einen Dichter hält, lieber der Reflexionsprosa (zu den Merkmalen dieser Prosa, z.B. Denka, 2008). Die Straußsche Prosa unterscheidet sich von dem, was man im allgemeinen unter Prosa versteht, und sie ist tatsächlich mit der lyrischen Dichtung verwandt, vor allem zeichnet sie sich durch eine reichhaltige Metaphorik aus.

\section{Metaphern des Gedächtnisses}

Zahlreiche Metaphern des Gedächtnisses geben dem Text Herkunft sein Gepräge. Eine von ihnen - „Kristalle“ (H, S. 59) - darf als Variante der anderenorts vielfach benutzten und erkundeten Metapher von Fleck und Linie (Strauß, 1992, S. 70) betrachtet werden (zur Verwandtschaft mit dem Topos „Linie und Kugel“: Thomas, 2004, S. 190).

Du blickst in deine Frühe wie in die blaue Kugel des Magiers, betrachtest ein abgetrenntes, umschlossenes Weltlein. Es ist nicht alles organisch, nicht alles Folge von Auffächerung, Fortschritt und Wachstum, was sich Leben nennt. Es bilden sich auch Kristalle, die sammeln und bündeln Strahlen und sind beständiger als Zeitspuren. (H, S. 59) [Hervorhebungen A.D.]

„Kugel“ und „Kristalle“ sind nicht linear. Es gibt nicht einen Punkt, von dem aus die Linien fächerartig auseinanderstreben und so zu verfolgen wären. „Fortschritt“ und „Wachstum“, die durch Ideologie, Politik und Wirtschaft geprägten Begriffe, geben keineswegs wieder, was der Mensch als sein Leben empfindet. Die Vergangenheit ist hermetisch, unzugänglich, ein Ding an sich, ein abgeschlossenes Dasein, eine andere Realität. Ein typischer Strauß-Widerspruch gegen die teleologische Weltauffassung, der auch mit seinem Zeit-Begriff zusammenhängt und den Strauß bereits in Der junge Mann (1984) formulierte:

\footnotetext{
Was nun das Element der Zeit betrifft, so muß uns auch hier eine weitere Wahrnehmung, ein mehrfaches Bewußtsein vor den einförmigen und zwanghaften Regimen des Fortschritts, der Utopie, vor jeder sogenannten Zukunft schützen. Dazu brauchen wir andere Uhren, das ist wahr, Rückkoppelungswerke, welche uns befreien von dem alten, sturen Vorwärts-Zeiger-Sinn. Wir brauchen Schaltkreise, die zwischen dem Einst und Jetzt geschlossen sind, wir brauchen schließlich die lebendige Eintracht von Tag und Traum, von adlergleichem Sachverstand und gefügigem Schlafwandel. (S. 11) [Hervorhebung A.D.]
} 
Auch die von einigen Kritikern zitierte Metapher des „Briefbeschwerers“ (H, S. 94-96) erscheint als Variante der Denkfigur Fleck und Linie, wobei der Briefbeschwerer offensichtlich dem Fleck entspricht: „ein Cabochon, Rundstein aus Onyx“ (H, S. 94), „,vom Umfang einer Fliegenpilzhaube, einer Quallenglocke“ (ebd.), „Es ist ein Stein, nichts als ein bearbeiteter Stein.“ (H, S. 95), „Ich verstehe nicht, wie ein solcher Stein eine quadratische, scharfkantige, eckige, spitze Form besitzen kann, obschon dies oft genug der Fall ist" (ebd.). Der Briefbeschwerer soll die Vergangenheit bändigen, den Zeugnissen der Vergangenheit, „den Schriftstücken, Briefen, Zetteln, Rechnungen“ (H, S. 94) gerecht werden. Eine Vergangenheit, die sich auch linear anordnen ließe, in einem Ordner, in einer Kartei, in einer elektronisch angelegten Liste eines Computerprogramms, wird auf eine einfache, primitive, geradezu atavistische Weise zusammengehalten.

$\mathrm{Da} ß$ der Stein so fest, die Zettel so lose... Eines Tages wird er nicht mehr zu heben sein von der Notiz, dem Zeitungsausschnitt, der verfallenen Opernkarte, der Adresse eines Immobilienmaklers, den Fitzeln, den längst überholten Memoranden. Übrig blieb: Vermischtes. (H, S. 96)

Diese Gegenüberstellung der geordneten Welt, die eine teleologische Weltauffassung mit einschließt (auf der einen Seite), und der Brüchigkeit des Vergangenen, der Erinnerung (auf der anderen Seite), wird von einem ästhetischen, gar ästhetizistischen Programm gestützt. Der Briefbeschwerer wird für seine eingeschränkte Zweckmäßigkeit gelobt, als ein Ding, das zu keinem anderen Teil gehört, „es war nirgends kombinierbar- oder addierbar. Etwas Lebloses (...), wie alle Dinge, die kein Teil sind, deren Zweck geringer wiegt als die Form, die mithin in das ästhetische Feld des Menschen eingedrungen sind“" (H., S. 94-95).

In einem Rückblick auf sein Leben entfaltet Botho Strauß subtil die Metapher des „Leinpfades“ (H, S. 92), wohl in der Erinnerung an die Topographie des an der Lahn gelegenen Bad Ems.

Dies Dahinziehen in Motiven und die fortschrittlose Bewegung in kurzen Sprüngen vor und zurück (...) lassen insgesamt auf ein Leben von geringer Tatkraft und großer Saumseligkeit schließen. Der Saum, ja, es war der schmale Lein- oder Treidelpfad, der zuerst an der Lahn und später am Fluß der Ereignisse entlangführte. (H, S. 92)

Der Lebensweg, der sich dem Autor als Leinpfad an der Lahn eingeprägt hatte, erscheint als etwas, was den Weg weist, aber auch den Widerstand, das ,retardierende Moment ${ }^{`}$ mit einschließt. Botho Strau $\aleph^{‘}$ Lebensphilosophie hieß „fortschrittlose Bewegung in kurzen Sprüngen“, „geringe Tatkraft“, „Saumseligkeit“. Dieses Programm entzieht sich der Totalität des Teleologischen. Ein solches Vorhaben war offensichtlich von vornherein zum Scheitern verurteilt: „Dennoch will es mir scheinen, als hätte ich aus tausend Veränderungen, Umstürzen und Digressionen nichts anderes als die Spur der Nachfolge bloßgelegt, das Relief einer Wiederholung, die Oberfläche einer tiefen Prägung gereinigt und geputzt.“ (H, S. 92) 
Was in Der junge Mann (1984) noch als optimistisches Programm erschien: es ließe sich in einem Augenblick die ganze gegenwärtige Welt auf Dauer einschließen (zirkulare, konzentrische Zeitauffassung), wird aus der Perspektive des alternden Autors korrigiert. Resignation des Autors, Bekundung des Unvermögens? Wohl nicht ganz, wenn man auf die Form des Buches schaut. Im Buch gibt es wenig Lineares - Biographisches zum Vater, zur Mutter, zum Autor selbst kann man sich höchstens aus Splittern zusammensetzen. Man muss Geduld üben und genau erkunden. Mitunter gewinnt es Züge einer kriminalistischen Spurensuche - und vor allem ist es ein ästhetisches Vergnügen.

\section{Die Liebe zum Detail}

Bereits in seinem Text Das Partikular (2000) hat Botho Strauß die Bevorzugung des Partikularen gegenüber dem Universellen zu seinem poetischen Grundsatz erhoben:

Aber was sieht das Auge des Todes von mir? Nur mein mühseliges Entgegenkommen. Nichts als ablaufende Zeit. Gesehen aber, wahrhaftig gesehen werde ich durch Sein Partikular. Das Partikular, durch das der Ewige uns sucht, erfaßt uns ohne zeitliches Brimborium, ohne geschichtliche Ergänzung und Verfälschung. (Strauß, 2002, S. 84)

Strauß versuchte literarisch das Prinzip des Malers Wols (Taschismus, Informel) umzusetzen, der den Akt der Wahrnehmung (flow) selbst malte. Wie viele andere Bücher von Strauß, besteht auch Das Partikular aus Skizzen, Geschichten, Erzählungen und Fragmenten. Das Schreibprinzip entsprach also dem ,Beflecken ${ }^{`}$ der Malfläche, was den psychischen Zustand des Künstlers und des Dichters widerspiegeln sollte. Entscheidend war dabei die Anfechtung des Zusammenhängenden, des Ganzen. Was zählte, waren die Details „die feinsten Ausfransungen der Beiläufigkeit“" (Strauß, 2002, S. 199).

Dasselbe Prinzip liegt dem Text Herkunft zugrunde. Ein Detail wird in den Blick genommen, um etwas mehr über das Ganze auszusagen, exemplarisch steht hier z.B. die Hand des Vaters. Es mag verwundern, dass sich ein Autor vier Seiten lang über die Hände seines Vaters ausbreiten kann (H, S. 36-40). Botho Strauß gelingt es auf seine ganz eigene Weise. Wenn das Buch eine Hommage an den Vater ist, so sind diese vier Seiten eine Hommage an die Hände des Vaters. Jedes Detail ist wichtig: ,in der rechten Handinnenfläche, dicht neben der Hauptsehne, eine höckrige Knöchelbildung“ (H, S. 38), „die Rundung zwischen Daumen und Zeigefinger“ (H, S. 39). Es beginnt mit einer Schilderung von Alltagsgesten:

Die Hand war allenfalls nicht besonders geschickt, wenn es darum ging, an den Apparaten zum technischen Gebrauch etwas einzustellen oder zu fügen. Es fiel ihr schwer, und sie zitterte leicht; unwirsch, nicht ängstlich zitterte sie, wenn ein Radio oder ein Plattenspieler bedient werden mußte. 
Ich kenne sie nicht als Faust, und ich kenne nicht seine zehn Finger ineinander gefaltet oder verwunden. Wohl die Fingerspitzen leicht zusammengesteckt, wenn beide Hände über dem Bauch lagen beim Mittagsschlaf - so wie ich sie zum letzten Mal sah, auf dem Totenbett. (H, S. 36)

Folgerichtig wird auf bestimmte Verhaltensschemata und auf das Feld des sozialen Handelns im weiteren Sinne verwiesen. Es folgen Aussprüche, die in ihrer Totalität beinahe als Aphorismen funktionieren könnten: „Die Hand hat mich gestraft und liebkost; sie hat mir die ersten Blumen gewiesen und die erste Zeile im Buch.“" (H, S. 36) Die Hand steht hier als Pars pro to to für den Vater selbst. Assoziationen wie etwa „die Hand, die dich füttert“, „die Hand Gottes“ stellen sich ein. In einem gewissen Sinne spiegelt die aphoristische Verdichtung das patriarchalisch geordnete Weltbild aus der Zeit vor der Studentenrevolte von 1968 wider (siehe z.B. Dittberner, 1998).

Mit der Feststellung „Die Hand legte Wert auf formgerechte Begrüßung.“ (H, S. 37) geht Strauß zum althergebrachten Zeremoniell und zu den eigenwilligen Ritualen des Vaters über. Die Hand wird „schneidend“ geboten, wenn ein Schulkamerad ermahnt wird, sich zur Begrüßung zu erheben (H, S. 37-38). Die Hände sind es, die den Vater in seinen Lebensrollen charakterisieren, bei der Arbeit (Umgang mit pharmazeutischen Gegenständen), bei der ,Handhabunge von Kultur (Schreiben, Klavierspiel). Dabei geht es immer um ein wechselseitiges Spiel von Gebärde (als Geste) und gesellschaftlicher Norm: die Norm bestimmt die Gebärde, die Gebärde bestimmt die Norm. Die Gebärde erreicht somit ihre zweite, konnotative Bedeutung, sie wird zum Habitus.

Bei der Wohnungsentrümpelung sieht der Erzähler „,das rote Dreieck der Dugena-Uhr, auf das [er] aus [seinem] Kinderzimmer in Richtung Kursaal hinunterblickte (H, S. 68)“. Strauß zeigt, wie bestimmte, kleine, scheinbar nebensächliche Dinge Spuren im Gedächtnis hinterlassen und gar Gewohnheiten in Form von Skripts prägen. Die Gewohnheit überdauert alle Ortswechsel: Immer wieder kommt es vor, dass ,ich noch heute im Traum ans Fenster renne, um auf meine Uhr zu sehen, die über dem Juweliergeschäft von der Wand abstand (H, S. 68)“. Ein Erinnerungsbild, das in seiner Schlichtheit als universale Erfahrung nachvollziehbar wird. Plötzlich fehlt die Uhr, die nach dem Umzug an einen neuen Wohnort nicht mehr vom Fenster aus zu erblicken ist. Damit wird ersichtlich, wodurch Straußens so vielfach gestaltete Reflexionen zum Zeitbegriff (teleologische vs. konzentrische Zeitauffassung) womöglich an der Quelle beflügelt worden waren. Als primäre Zeiterfahrung gilt „das rote Dreieck der Dugena-Uhr“. Und nun stellt sich die Erinnerung ein: „Unter der Uhr ging der kleine, plattfüßige Dr. Ulmer, der untreue Notar, Geliebter der Inhaberin des Schuhladens.“ (H, S. 68). Hier konstruiert Strauß einen der schönsten Momente des Buches:

Er blickte von der Straße hinauf zur Uhr, und beinahe hätten wir, Träumender und Traumfigur, einander erblickt, über Jahrzehnte und sogar über seinen Tod hinweg, wäre nicht mein Blick von 
oben und seiner von unten getrennt worden von ebendiesem elektrischen Zeitmesser-Dreieck, darin das Zifferblatt mit dem rundlaufenden Zeiger. (H, S. 68)

Der alte Bekannte, über den man im Städtchen wegen seiner Untreue tratschte, wird beinahe als mythische Figur verewigt, findet Eingang in Literatur, wie auch sonst viele andere Helden, Autoren aus Straußens Universum. Es ist ein Beispiel für die Verewigung des Augenblicks. Die Liebe zum Detail wird der Erinnerungspoetik untergeordnet.

So auch ein aus dem Radio wahrgenommenes Musikstück: „Capriccio italien, das Triviale leitet wie nichts anderes widerstandslos den Strom der Erinnerung. Musik überhaupt ruft ohne jedes Gedenken ein Damals in Echtzeit herauf." (H, S. 70) Schon befindet sich das Subjekt in seinem Kinderzimmer, ,den Taktschlag übend, Partituren lesend (...), dabei waren die Wände noch mit Star-Fotos beklebt, Übergangszeit von Rudolf Prack zu Arturo Toscanini, ,Star-Revue', ,Film-Revue' hießen die Magazine, neben Winnie Markus Bruno Walter. Später folgte ,Das Schönste', eine europäische Kulturzeitschrift, ganzseitige Bilder von Caravaggio, Feininger, Klee. Zur Zeit der ersten Lieben war das, denn sie hoben den Geschmack..." (H, S. 70) Auch hier gilt das Prinzip - vom Detail zum Ganzen, mag das Ganze auch noch so bruchstückhaft und unsystematisiert erscheinen. Auslöser sind einige Takte einer Musik, und schon wird ein stream of consciousness zur adoleszenten Erfahrung des Subjekts mit Kultur freigesetzt, formen sich Passagen eines Bildungsweges, in Sprüngen zwischen Filmund Schauspielkunst (Rudolf Prack, Winnie Markus), Musik (Dirigent Arturo Toscanini, Dirigent und Komponist Bruno Walter) und Malerei (Caravaggio, Feininger, Klee). So erfahren wird etwas über das Kinderzimmer in Bad Ems und über die Kulturerzeugnisse, die den jungen Botho Strauß geprägt haben. Die Methode setzt beim Detail an und erhebt keinen Anspruch auf einen ,totalen ${ }^{`}$ Blick. Die persönliche Erinnerung unterscheidet sich insofern vom professionellen Blick des Historikers auf die Vergangenheit: „Sie sehen mit ihren jeweils aktuellen methodischen Klugheiten, ihrem habituellem Besserwissen unvermeidlich herab auf die frühere Epoche, die sie untersuchen. Anachronistisches Wissen ist der Fluch ihrer Profession. Der Lebende hingegen wird von Gelebtem katechisiert." (H, S. 71) Vermitteltes versus unvermitteltes Anschauen - ein Wissenschaftler untersucht, ein Lebender lebt!

\section{Misanthropie als Elitenbewusstsein}

Botho Strauß' Konstrukt der eigenen Autorschaft trägt seit einigen Jahrzehnten den Hauch des Elitären (Essenberg, 2004). Dies gilt auch für die Figuren, die er in seiner Reflexionsprosa beschreibt (manchmal sind das reale Gestalten, Autoren, Dichter, Künstler, u.a. Georg Büchner, George Steiner, Rudolf Borchardt, Robinson Jeffers, Nicolás Gómez Dávilla etc). In anderen Fällen sind es abstrakte Figuren, 
die sich vor allem durch ihr Außenseitertum auszeichnen, hier exemplarisch, der „Rechte“, der „Reaktionär“, der „Außenseiter-Heros“, der „Dichter“, der „,mutige Einsiedler“, der „Nicht-Einverstandene“, der „Einzelgänger“ (Denka, 2013, S. 292-294). Symptomatisch ist auch die Figur des Antihumanisten, die vor allem durch den amerikanischen Dramaturgen und Dichter Robinson Jeffers, verkörpert wird, dem Strauß den Abschnitt Jeffers Akt in der seiner aphoristischen Prosa Fragmente der Undeutlichkeit (1989) widmete: „Robinson Jeffers, Dichter der amerikanischen Westküste, lebte von 1887 bis 1962. Mit seiner Frau Una verließ er in jungen Jahren die Städte und siedelte abgewandt auf einer Klippe vor dem pazifischen Ozean“ (Strauß, 1989, S. 9). Jeffers zeichnete sich durch seine Skepsis gegenüber der Demokratie (hier vor allem der Roosevelt-Demokratie) und seine distanzierte Haltung gegenüber den Menschen aus: „Zwei drei Menschen an einem Tag, und er war für den Rest des Monats mit der menschlichen Rasse zerfallen" (Strauß, 1989, S. 13). Es wäre als seltsam anzusehen, dass ein Autor im Jahre der Wende 1989, des Sieges der Demokratie, einen Antidemokraten und Menschenhasser zum Helden macht, hieße der Autor nicht Botho Strauß, der einige Jahre später im Anschwellenden Bocksgesang (1993) seine Thesen gegen die liberale Demokratie vorbrachte.

Passt Robinson Jeffers zu Straußens widerspenstigen Helden, so wird nach dem Portrait des Vaters in Herkunft zudem ersichtlich, wie sehr der eigene Vater in diese Reihe der Ahnen gehört. Auch er ist ein Außenseiter-Heros, den Strauß anstelle des seines Erachtens obsoleten linksliberalen Intellektuellen in seinem Bocksgesang als Typus heraufbeschwört. War der Vater vielleicht ein Prototyp? Mit gutmütiger Ironie, doch ebenso mit ernsten Tönen versucht Strauß, dieses Heroische bereits an der Körperpflege festzumachen: „Die Morgentoilette war das unverzichtbare Zeremoniell einer häuslich-heroischen Selbstbehauptung. Es ging darum, Figur zu machen, sich abzuheben von den gewöhnlichen Zeitgenossen, die sich vernachlässigten.“ (H, S. 9)

Dann folgen Thesen von ganz anderem Gewicht, Schilderungen, die dem Vater ein Flair des Erhabenen verleihen: „Ein Mann war er. Ein einzelner nach altem Ibsen-Format. Ein Mann ist, wer zu lieben und zu hassen vermag. Kein Anwalt des Sowohl-als-auch. Ein Volksverächter mit törichten Verblendungen“ (H, S. 17). Welche der Gestalten von Ibsen (siehe auch H, S. 83) vor allem zum Vater passen soll, Gregers Werle aus Die Wildente, Peer Gynt oder Arnold Rubek aus Wenn wir Toten erwachen, bleibt dahingestellt. Jedenfalls erscheint der Vater als ein kompromissloser Mann, ein Individualist, der nicht ganz an die Gesellschaft angepasst ist, ein Konservativer, ein Reaktionärer in gewisser Hinsicht, aber im ,Dritten Reich 'offensichtlich hartnäckig genug, um nicht zum Mitläufer im Namen einer verbrecherischen Ideologie zu werden. Wolfgang Büscher bemerkt in seiner Rezension:

Dieser Typus ist ausgestorben, in der Nachkriegszeit bevölkerte er die mittleren Milieus der Bundesrepublik. (...) Diese Männer waren oft keine Nazis gewesen - die Nazis erwiesen sich oft als die Flexibleren, die im Strom der neuen Zeit besser schwammen als solche wie der zur Geschmeidigkeit so schlecht begabte Vater Strauß, kein Nazi auch er. (Büscher, 2014) 
Dennoch betont Strauß, dass die Misanthropie des Vaters erst recht spät sich entwickelt habe, als bestimmte Entwicklungsphase und Folge gewisser Erfahrungen (Verwundung im Ersten Weltkrieg, Verzicht auf das Medizinstudium, Verhaftung und Enteignung in der SBZ). Früher hatte er als Pharmavertreter und Apotheker rege Kontakte gepflegt, sein Lieblingsspruch war ein Satz aus dem Tasso gewesen: „es bildet ein Talent sich in der Stille, sich ein Charakter in dem Strom der Welt"“ (H, S. 29). Einmal heißt es „seine spätere Misanthropie“ (H, S. 31), ein anderes Mal „seine freimütige Misanthropie“ (H, S. 83). Immer eingeschränkt, abgeschwächt. Auf das Ganze bezogen, verkehrt sich die Misanthropie in ihr Gegenteil, wird beinahe zu einem menschenfreundlichen Prinzip.

Schließlich kommt auch der von Strauß in seinem übrigen Werk vielfach benutzte konservative Topos des „Aushaltens auf dem verlorenem Posten“, den man u.a. auf Ernst Jünger zurückführen könnte (Thomas, 2004, S. 145-146), zum Tragen:

Er ging ungebeugt jeden Morgen, schritt fest und gerade durch den Kurpark. Schöpfte frische Luft, bevor er sich an den Schreibtisch setzte und an seine verdammt schlecht bezahlte Arbeit machte. Es war ihm eine Menge schiefgegangen. Aber das brachte seine Figur nicht durcheinander. Er hätte auch aufgeben können, saufen, seine Familie zerstören. Wie nebenan der entlassene Major, den man zweimal die Woche aus der Gosse nach Hause trug. (H, S. 77)

Mag man auch in Herkunft die ideologisch motivierten Thesen, die vermeintlich reaktionären oder gar nationalen Sticheleien aus anderen Texten erkennen (den Bocksgesang eingeschlossen), und mag man auch Prototypen der Gleichgesinnten entdecken, so kann dies doch nicht darüber hinwegtäuschen, dass es sich um einen schönen, persönlichen und privaten Text handelt. Eine späte Liebeserklärung an den Vater, den der Autor zu Lebzeiten nicht genug zu lieben und zu schätzen vermochte, gegen den er rebellierte und den er manchmal hasste. Der Text ist autobiographisch, am Einzelfall orientiert und dennoch umfassend und so universell wie die verschiedenen Facetten der Vaterschaft, die Erfahrung, Vater zu sein, einen Vater zu haben, den Vater verloren zu haben und ihn zu vermissen. Es ist ein Text über das späte Hineinwachsen in den Tod des Vaters, über das späte Erkennen der Vaterrolle und ihren Beitrag zur Bildung vor allem der männlichen Identität. Ein Text, der die in den letzten Jahrzehnten von verschiedenen ideologischen Trends als anrüchig angesehene, geringgeschätzte und gar abservierte Figur des Vaters in einem neuen Licht erkennen lässt. Ein Korrektiv sozusagen. Und überdies eine fein gearbeitete Reflexionsprosa, über die man nur in höchsten Tönen sprechen kann.

\section{Bibliographie}

Arend, H. (2014). Die Wiederkehr des Autors. Der Dichter Botho Strauß schreibt über seine Heimat und seine Herkunft. Literaturkritik.de. Rezensionsforum. Abgerufen von http://literaturkritik.de/public/ rezension.php?rez_id=19842 
Büscher, W. (2014). Glück und Glas, wie leicht bricht das [Besprechung von Botho Strauß Herkunft] Die Welt [online]. Abgerufen von http://www.welt.de/kultur/literarischewelt/article 132709147/Glueckund-Glas-wie-leicht-bricht-das.html

Denka, A. (2008). Uckermark - miejsce magiczne w duchowej autobiografii Botho Straußa Die Fehler des Kopisten. In G. Moroz \& M. Ossowski (Hrsg.), Miejsca magiczne w literaturze anglo- $i$ niemieckojęzycznej (S.123-138). Olecko: Wydawnictwo Wszechnicy Mazurskiej.

Denka, A. (2013). Skandal oder Engagement? Eine systemtheoretische Untersuchung zu Peter Handke und Botho Strauß nach 1989. Poznań: Wydawnictwo Naukowe UAM.

Dittberner, H. (1998). Die Inthronisation der Väterwelt. Zu Botho Strauß und Peter Handke. Text+Kritik. Zeitschrift für Literatur, H. 81, 3-9.

Eckhoff, J. (1999). Der junge Botho Strauß. Literarische Sprache im Zeitalter der Medien. Tübingen: Max Niemeyer.

Essenberg, O. van (2004). Kulturpessimismus und Elitebewusstsein. Zu Texten von Peter Handke, Heiner Müller und Botho Strauß. Marburg: Tectum.

Hage, V. (1987). Schreiben ist eine Séance. Begegnungen mit Botho Strauß (1980 und 1986). In. M. Radix (Hrsg.), Strauß lesen (S. 188-216). München: Carl Hanser.

Hage, V. (2014). Der Vater als Bürde [Besprechung von Botho Strauß Herkunft]. Der Spiegel, 40, 142.

Mangold, I. (2014). Die Verteidigung der Einst-Welt [Besprechung von Botho Strauß Herkunft]. Die Zeit - Online. Abgerufen von http://pdf.zeit.de/2014/41/botho-strauss-herkunft-roman.pdf

Pabst, R. (2014). Dieser Herr wohnt hier nicht mehr. Lokaltermin in Bad Ems: Was die kleine Kurstadt mit der großen Vergangenheit über die Herkunft von Botho Strauß verrät. Frankfurter Allgemeine Zeitung [online]. Abgerufen von http://www.faz.net/aktuell/feuilleton/buecher/autoren/auf-den-spurenvon-botho-strauss-kurstadt-bad-ems-13335720.html

Sill, O. (2001). Literatur in der funktional differenzierten Gesellschaft. Systemtheoretische Perspektiven auf ein komplexes Phänomen. Wiesbaden: Westdeutscher Verlag.

Strauß, B. (1984). Der junge Mann. München: Carl Hanser.

Strauß, B. (1989). Fragmente der Undeutlichkeit. München: Carl Hanser.

Strauß, B. (1992). Beginnlosigkeit. Betrachtungen über Fleck und Linie. München: Carl Hanser.

Strauß, B. (1993). Anschwellender Bocksgesang. Der Spiegel, 6, 202-207.

Strauß, B. (1997). Die Fehler des Kopisten. München: Carl Hanser.

Strauß, B. (2002). Das Partikular. München: DTV.

Strauß, B. (2014). Herkunft. München: Carl Hanser (Sigle, H).

Thomas, N. (2004). ,Der Aufstand gegen die sekundäre Welt' - Botho Strauß und die ,Konservative Revolution'. Würzburg: Königshausen \& Neumann.

Wikipedia (2016). Eduard Strauss [Stichwort]. Wikipedia.de. Abgerufen von https://de.wikipedia.org/ wiki/Eduard_Strauss 